\title{
Self-assembly of 2,3-dihydroxycholestane steroids into supramolecular organogels as a soft template for the in-situ generation of silicate nanomaterials
}

\author{
Valeria C. Edelsztein, Andrea S. Mac Cormack, Matías Ciarlantini \\ and Pablo H. Di Chenna*
}

\section{Full Research Paper}

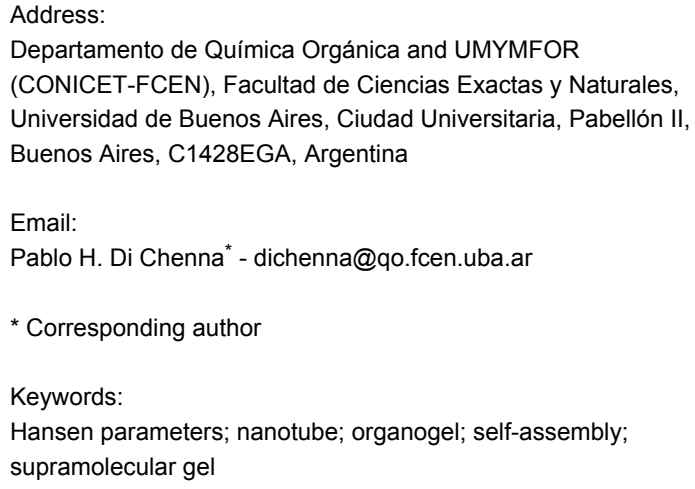

\author{
Beilstein J. Org. Chem. 2013, 9, 1826-1836. \\ doi:10.3762/bjoc. 9.213 \\ Received: 15 May 2013 \\ Accepted: 12 August 2013 \\ Published: 09 September 2013 \\ Associate Editor: S. C. Zimmerman \\ (c) 2013 Edelsztein et al; licensee Beilstein-Institut. \\ License and terms: see end of document.
}

\begin{abstract}
Supramolecular gels are an important and interesting class of soft materials that show great potential for many applications. Most of them have been discovered serendipitously, and understanding the supramolecular self-assembly that leads to the formation of the gel superstructure is the key to the directed design of new organogels. We report herein the organogelating property of four stereoisomers of the simple steroid 2,3-dihydroxycholestane. Only the isomer with the trans-diaxial hydroxy groups had the ability to gelate a broad variety of liquids and, thus, to be a super-organogelator for hydrocarbons. The scope of solvent gelation was analysed with regard to two solvent parameters, namely the Kamlet-Taft and the Hansen solubility parameters. The best correlation was observed with the Hansen approach that revealed the existence of two clear gelation zones. We propose a general model of self-assembly through multiple intermolecular hydrogen bonds between the 1,2-dihydroxy system, which is based on experimental data and computational simulations revealing the importance of the di-axial orientation of the hydroxy groups for the one-dimensional self-assembly. Under controlled conditions, the fibrillar superstructure of the organogel was successfully used as a template for the in-situ sol-gel polymerization of tetraethoxysilane and the further preparation of silica nanotubes. We propose that the driving forces for templating are hydrogen bonding and electrostatic interactions between the anionic silicate intermediate species and the self-assembled fibrillar network.
\end{abstract}

\section{Introduction}

Low molecular mass organogelators (LMOGs) have received increasing attention during the last two decades because of their unique properties and numerous potential applications in fields such as the stabilization of organic photochromatic materials, the templated synthesis of nanostructured and functional materials, the controlled release drugs systems, the capture of 
spilled pollutants in the environment, electrochemistry, lightharvesting materials and so on [1-6]. These small molecules self-assemble into regular supramolecular structures through non covalent interactions such as ion-ion, dipole-dipole, hydrogen bonding, $\pi-\pi$ stacking, van der Waals, host-guest, and ion coordination, and in so doing trap the solvent molecules in the supramolecular network to form supramolecular gels. The non-covalent nature of these interactions makes it possible for the supramolecular gel systems to achieve a reversible sol-gel phase transition by the simple application of an external stimulus. Intrinsically, supramolecular gels are thermosensitive and can be transformed reversibly to a fluid (sol) by heating. A small number of novel LMOGs, however, undergo a sol-gel transition as the temperature increases, which is called thermogelling [7]. Many other LMOG molecules form gels that are sensitive to other physical stimuli such as light, ultrasound or chemical stimuli [8-12]. A wide variety of structurally diverse molecules have the ability to form physical gels (e.g., saccharides, peptides, ureas, nucleobases, steroids, dendrimers, etc. [13]). Although a great effort has been made to investigate the structure-property relationships, it is still impossible to design a new LMOG de novo. For those reasons most of the known LMOGs have been discovered serendipitously. Nevertheless, with the knowledge gained about the mode of aggregation of LMOG molecules some of the structural features necessary for gelation are known. The presence of a supramolecular synthon to promote the one-dimensional (1D) selfassembly is a necessary feature in order to form the fibrillar entangled network that entraps the solvent [14]. The strongest and most important supramolecular synthons involve functional groups that possess a complementary donor-acceptor hydrogen bond motif, such as for instance amides, ureas, carbamates, saccharides, ammonium carboxylate salts, etc. A rod-like molecular shape is also a general structural requirement for steroid derived LMOGs because it allows a good face to face molecular contact to promote the one-dimensional growth. These concepts have been recently exploited to design new LMOGs $[15,16]$. Nevertheless, the presence of a supramolecular synthon in a molecule is a necessary but not a sufficient feature to become an organogelator. The formation of the gel involves a delicate balance of cooperative forces between the directional self-assembly that promotes the $1 \mathrm{D}$ aggregation and the solubility and insolubility in a given solvent, which is based on the specific interactions between solvent and gelator molecules [17]. Numerous attempts have been made to correlate solvent parameters to gelation ability. The most promising technique was recently presented in the works of Bouteiller et al. and Rogers et al., in which they apply the Hansen solubility parameters (HSP) to evaluate the gelation behavior of LMOGs in different solvents $[18,19]$. The Kamlet-Taft solvatochromic parameters, which consider separately the hydrogen-bond donor (HBD, $\alpha$ ), hydrogen-bond acceptor (HBA, $\beta$ ), and polarizability $\left(\pi^{*}\right)$ properties as contributions to the overall solvent polarity had also been occasionally used to study solvent-gelator specific interactions $[20,21]$.

LMOGs based on cholesterol and bile acids offered the most versatile units on which to base the systematic design of functional LMOGs for the gelation of organic solvents. Neither cholesterol nor cholestanol are gelator molecules, and although a variety of steroid derivatives has been analyzed over the years only a few simple analogues are known to be organogelators [22]. The steroidal LMOGs usually have substituents attached to the $3 \beta-\mathrm{OH}$ of the cholestane A ring and synthetic variations at the steroidal skeleton are scarce. Cholesterol-based LMOGs build mesophases in which steroid-steroid stacking is controlled by van der Waals forces. These interactions, including the additional intermolecular contacts from the pending moieties linked at C-3 (usually hydrogen bond and $\pi-\pi$ stacking), lead to a primarily one-dimensional long-range growth and finally produce the interconnected 3D self-assembled fibrillar network (SAFIN), which traps the solvent and turns into a self-supporting gel. There are several types of cholesterol-based LMOGs grafted on hydrophilic heads such as saccharides, chromophores, ligands, peptides, etc. The first study on rational syntheses of cholesterol derived organogels was made by Weiss et al. [23] on a family of molecules containing an aromatic moiety (A) connected to a steroidal group (S) through a linker (L). Since then a great number of ALS molecules have been discovered constituting the most systematically investigated family of LMOGs [24].

Recently we have reported a new steroid-based organogelator with a non-conventional bridged pregnane skeleton and its use as template for the preparation of nanotubes and fluorescent nanospheres of silica via the in-situ sol-gel polymerization of tetraethyl orthosilicate (TEOS) $[25,26]$. In our search for new LMOGs analogues with structural variations in the steroidal nucleus and new properties we prepared steroid $\mathbf{1}$ as a synthetic intermediate (Figure 1). This known steroid, which is widely used in medicinal chemistry as a precursor in the synthesis of cholesteryl derived bioactive analogues [27,28], gelated $n$-hexane during concentrating the fractions from a chromatographic column. Although this simple steroid has been synthesized for the first time decades ago, its gelation property has never been described in the literature.

In this paper, we report the broad scope and super-organogelating ability of steroid $\mathbf{1}$ and the use of their gels as template for the in situ polymerization of TEOS. A general self-assembly model with multiple intermolecular hydrogen bond interactions is proposed based on experimental and computational data. In 


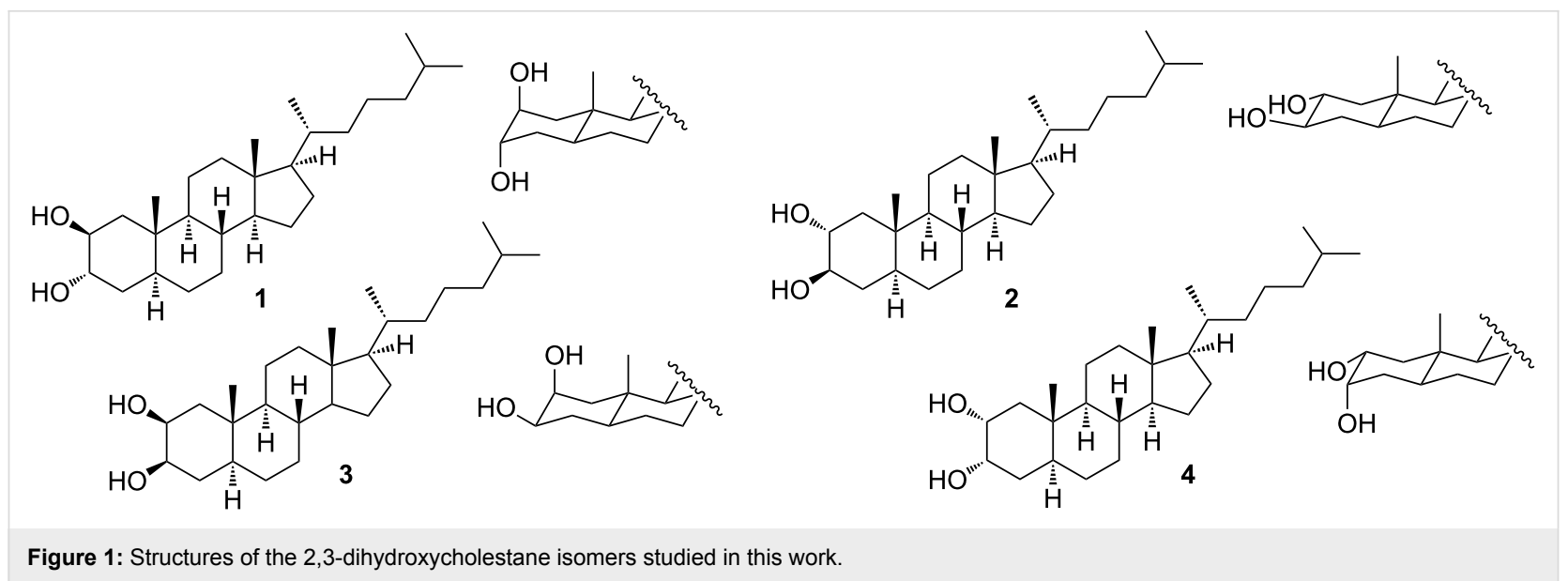

order to understand the role of the trans-diaxial orientation of the vicinal dihydroxy moiety we have also studied the organogelating properties of the stereoisomers $2-\mathbf{4}$ bearing equatorial hydroxy groups (Figure 1). The gelation ability of $\mathbf{1}$ is discussed in terms of the Hansen solubility parameters and Kamlet-Taft parameters based on its behavior in a set of 33 solvents. We also report on the sol-gel polymerization of TEOS carried out with gels of $\mathbf{1}$ demonstrating that templated silica nanotubes are obtained only under controlled conditions. Considering that the design of new LMOGs with predictable gelation properties is still a challenge nowadays, we consider that the trans-diaxial dihydroxy supramolecular synthon studied herein is a valuable contribution towards the development and design of new LMOGs molecules with potential applications.

\section{Results and Discussion}

As mentioned before, steroid 1 showed an excellent gelation ability in $n$-hexane during concentrating a solution on a rotatory evaporator. Preliminary qualitative tests with cyclohexane and dichloromethane also showed a good gelling ability. These remarkable properties for such a simple steroid molecule prompted us to study the gelation scope, morphology, mode of self-assembly and the potential use of the gels to prepare silica nanoparticles through a bottom-up approach.

\section{Gelation scope and thermal stability}

To assess the scope of the gelation ability of steroids 1-4 in a simple way, the test tube method was first used with 28 selected organic solvents ranging from hydrocarbons to alcohols (Table 1, entries 1-28). While steroids 2-4 were unable to gelate any of these solvents, steroid $\mathbf{1}$ showed a good to excellent gelation ability over a wide variety of solvents.

Given these results we directed our analysis towards the scope of solvent gelation of $\mathbf{1}$ by using the HSP approach described by Boutellier [18]. A tridimensional plot of the dispersive interac-

\begin{tabular}{|c|c|c|c|}
\hline entry & solvent & test $^{a}$ & $\mathrm{CCG}^{\mathrm{b}}(\mathrm{wt} \%)$ \\
\hline 1 & 1,2-dicloroethane & G & 1.4 \\
\hline 2 & 1,4-dioxane & G & 7.0 \\
\hline 3 & 1-hexanol & $S$ & - \\
\hline 4 & acetic acid & $S$ & - \\
\hline 5 & acetone & I & - \\
\hline 6 & acetonitrile & 1 & - \\
\hline 7 & aniline & G & 2,0 \\
\hline 8 & $\mathrm{CCl}_{4}$ & G & 0.8 \\
\hline 9 & chloroform & G & 2.5 \\
\hline 10 & pyridine & $S$ & - \\
\hline 11 & dichloromethane & $G$ & 0.8 \\
\hline 12 & DMF & $S$ & - \\
\hline 13 & DMSO & $G$ & 5.0 \\
\hline 14 & ethanol & 1 & - \\
\hline 15 & ethyl acetate & $S$ & - \\
\hline 16 & isopropyl ether & 1 & - \\
\hline 17 & methanol & I & - \\
\hline 18 & $n$-butanol & 1 & - \\
\hline 19 & $n$-heptane & TG & 0.06 \\
\hline 20 & $n$-hexane & TG & 0.06 \\
\hline 21 & cyclohexane & TG & 0.13 \\
\hline 22 & TEA & I & - \\
\hline 23 & TEOS & $S$ & - \\
\hline 24 & THF & $S$ & - \\
\hline 25 & toluene & G & 2.5 \\
\hline 26 & water & 1 & - \\
\hline 27 & xylene & G & 0.8 \\
\hline 28 & methyl acrylate & $G$ & 4.0 \\
\hline 29 & nitrobenzene & $G$ & 3.3 \\
\hline 30 & methylcyclohexane & TG & 0.16 \\
\hline 31 & decane & TG & 0.04 \\
\hline 32 & styrene & G & 2.5 \\
\hline 33 & o-dichlorobenzene & G & 5.0 \\
\hline
\end{tabular}

aG: gel, TG: turbid gel, S: soluble I: insoluble; ${ }^{b}$ critical concentration for gelation. 
tions $\left(\delta_{\mathrm{d}}\right)$, the dipolar-interactions $\left(\delta_{\mathrm{p}}\right)$ and the hydrogenbonding $\left(\delta_{\mathrm{H}}\right)$ parameters of LMOG 1 showed a complex correlation between the gelation ability and the HSP of the solvents (Figure 2, see Supporting Information File 1 for full data). Two gelation zones were clearly identified graphically. The first gelation space (zone A), with a cylindrical, almost linear profile, involves solvents with zero or very low $\delta_{\mathrm{H}}$ and $\delta_{\mathrm{p}}$ parameters and a $\delta_{\mathrm{d}}$ parameter between 14 and 18 (hydrocarbons). Gelation zone B includes polar, non-protic solvents with higher dispersive interaction parameters $\delta_{\mathrm{d}}$ (from 17.5 to 20 ). In contrast to the results obtained by Boutellier et al. [18], it was not possible to define a gelation sphere in either zone in this case. Nevertheless, in order to corroborate the tendency observed we chose a new set of solvents with HSP between the gelation spaces (Table 1, entries 29-33), all of them could be gelated by 1 showing that, although spherical spaces cannot be defined, prediction is possible by selecting solvents with HSP inside the gelation zones. Figure 2 qualitative shows that there is a good correlation between the HSP and the gelated solvents, pyridine and methyl acrylate are the only solvents that visible lay outside the corresponding zones. It is clear from the graphic analysis that the higher the $\delta_{\mathrm{H}}$, the higher the $\delta_{\mathrm{p}}$, of the gelated solvent with a limit of about 10 for the former. Non-gelled solvents are clustered in zones with high $\delta_{\mathrm{H}}$ and low $\delta_{\mathrm{d}}$. This makes sense, because a solvent, which acts as either a strong hydrogen-bond donor or a strong hydrogen-bond acceptor, will significantly interact with the hydroxy groups of $\mathbf{1}$ and thus impede the supramolecular self-assembly and the formation of the gel. This way it will prevent gelation or will make a higher concentration of gelator necessary. This effect is higher for solvents with smaller dispersive interaction parameters. For solvents in zone A steroid $\mathbf{1}$ is a supergelator with critical concentrations for gelation (CCG) below $0.1 \mathrm{wt} \%$ for linear hydrocarbons (entries 19, 20 and 31) and of about $1 \%$ for cyclic hydrocarbons and carbon tetrachloride (entries 8, 21 and 30). The second zone comprises aromatic and polar solvents bearing nitrogen or oxygen atoms with CCG values between 2 and 7 wt $\%$. The presence of two gelation zones is indicative of a difference in the molecular packing of the gel fibers and will be discussed in the following section.

Next we considered the Kamlet-Taft parameters for the tested solvents and compared these results to the Hansen approach [29]. The 3D plot did not show clear zones of gelation (Figure 2b) although it is evident from the analysis that solvents with very high $\alpha$ parameters ( $\alpha>0.5$, such as alcohols, water and formic acid) cannot be gelated. The same tendency is observed for the $\beta$ parameter but with some exceptions, such as DMSO, which is a very strong hydrogen-bond acceptor but still capable of forming gels. The effect of $\pi^{*}$ seems to be less important since solvents with both high and low polarizability can be gelled (see $\pi^{*}$-vs- $\alpha$ and $\pi^{*}$-vs- $\beta$ plots in Supporting Information File 1). We assume that the bad correlation between the Kamlet-Taft parameters and the gelation behavior of LMOG 1 is connected to the solvatochromic origin of the scale [29]. The Hansen solubility parameters with a scale based on solubility seems to be more suitable for solvent-gelation analysis.

All gels were stable, thermoreversible and non-thixotropic. Gels from hydrocarbons were turbid, depending on the concentration of gelator, indicating a low solubility. To estimate the relationship between thermal stability, concentration of gelator, and solvent we studied the variation of the gelation temperature $(\mathrm{Tg})$ with the concentration of $\mathbf{1}$ in gels of cyclohexane, dichloromethane, carbon tetrachloride, dioxane, aniline and nitrobenzene. Tube inversion experiments were performed to measure $T_{\mathrm{g}}$. This method was selected because of its simplicity and
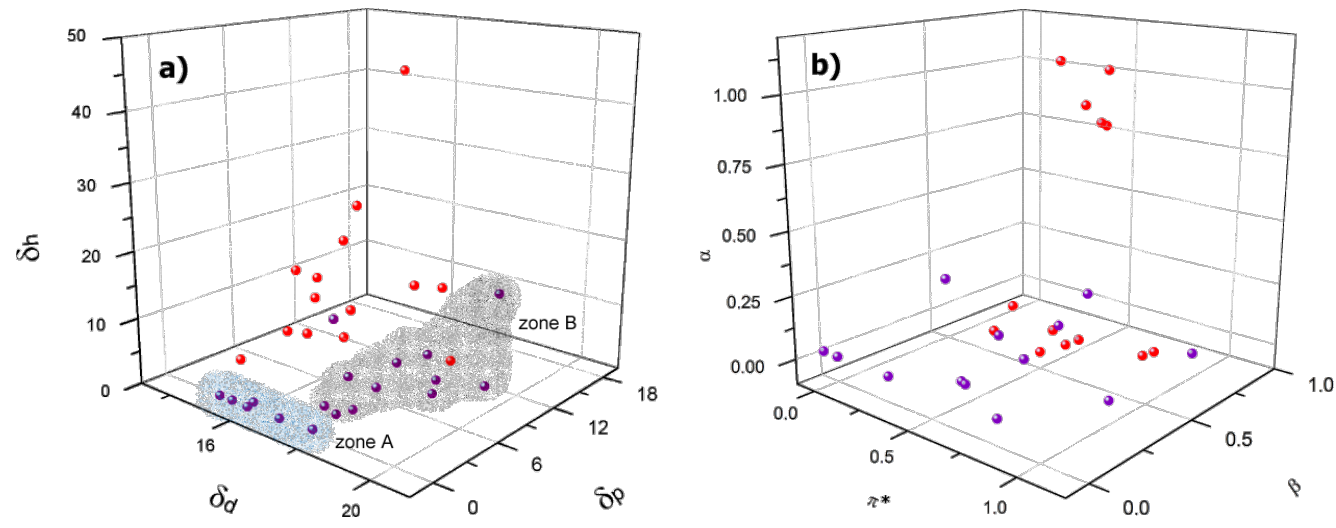

Figure 2: 3D plots for LMOG 1 and solvent parameters of the tested solvents a) Hansen solubility parameters ( $\delta_{d}$ dispersive interactions, $\delta_{p}$ dipolar interactions, $\delta_{H}$ hydrogen bonding) and b) Kamlet-Taft parameters ( $\alpha$ hydrogen bond donor, $\beta$ hydrogen bond acceptor, $\pi^{*}$ polarizability). Purple: gelated solvents. Red: non-gelated solvents. (For more details see Table S1 in Supporting Information File 1.) 
widespread use in the field of gel-phase materials. Typically, as the concentration of $\mathbf{1}$ was increased, $T_{\mathrm{g}}$ also increased until a plateau region was reached. Cyclohexane gave the most stable gel with a $T_{\mathrm{g}}$ of $64{ }^{\circ} \mathrm{C}$ at the CCG, and a maximum $T_{\mathrm{g}}$ value of $99{ }^{\circ} \mathrm{C}$ for a $2-w t-\%$ gel (Figure 3). Carbon tetrachloride and dichloromethane reached the plateau region at the same concentration, but with $T_{\mathrm{g}}$ values of 75 and $45^{\circ} \mathrm{C}$, respectively, which shows that more polar solvents gives thermally less stable gels. On the other hand, dioxane, nitrobenzene and aniline, capable

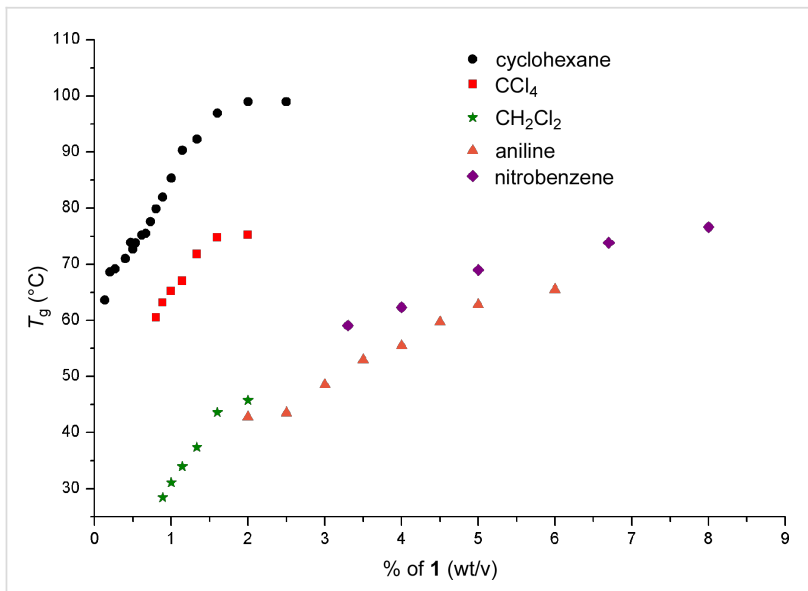

Figure 3: $\mathrm{Tg}$-vs-concentration plots for gels of 1. of hydrogen bonding, had a maximum $T_{\mathrm{g}}$ of $72{ }^{\circ} \mathrm{C}, 77^{\circ} \mathrm{C}$ and $66^{\circ} \mathrm{C}$, respectively, but at considerably higher concentrations of 26, 6 and $8 \mathrm{wt} \%$ (see Supporting Information File 1). LMOG 1 could also selectively gelate the organic layer from a water/ organic solvent mixture after a heating-cooling process.

\section{Morphology and self-assembly}

The FTIR spectra of the gel and the solution of LMOG 1 provided evidence that the interactions leading to self-assembly is primarily hydrogen bonding between the hydroxy groups. In dichloromethane (DCM) solution, at a concentration of LMOG 1 below the CCG, a broad band was observed at $v_{\mathrm{O}-\mathrm{H}}=3608.2 \mathrm{~cm}^{-1}(\mathrm{O}-\mathrm{H}$ stretching). In the gel state this band was widened and shifted to $3363.3 \mathrm{~cm}^{-1}$, which is typical for an intermolecular hydroxy hydrogen-bond. The FTIR spectrum of the gel still showed the band of free hydroxy groups at $v_{\mathrm{O}-\mathrm{H}}=3610.1 \mathrm{~cm}^{-1}$ and may be associated with molecules of LMOG 1 in the liquid-like solution phase trapped within the SAFIN (see Supporting Information File 1 for IR spectra) [17].

The microscopic morphology of the xerogel of 1 from DCM, $n$-hexane and dioxane was analyzed by SEM. The images showed an entangled fibrillar network for all solvents. Particularly the images of the dichloromethane xerogel (Figure 4a and Figure 4b) showed left handed helical fibers with a fiber width
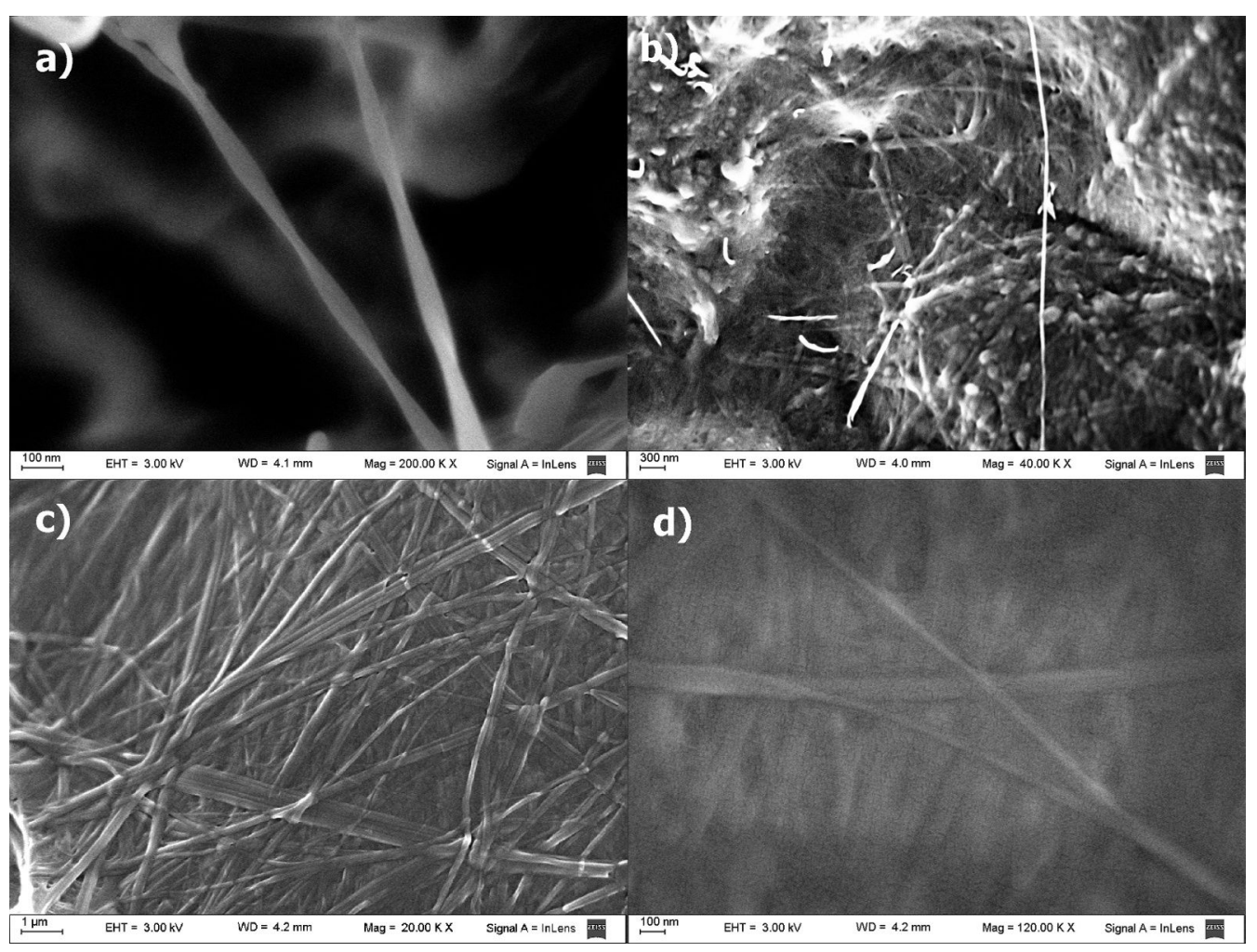

Figure 4: SEM images of xerogels from $a, b)$ dichloromethane, and c,d) from dioxane. 
ranging from 20 to $75 \mathrm{~nm}$, and a helicoidal period of about $750 \mathrm{~nm}$. The helical shape is clearly observed in the isolated fibers (Figure 4a), but these are difficult to find in the bulk due to the collapsed fibrillar network. For $n$-hexane and dioxane simple straight entangled fibers were observed with a minimum width of $20 \mathrm{~nm}$. In all cases fibers with lengths of up to several micrometers were clearly visible. SEM images of the xerogel from dioxane showed a tighter SAFIN compared to $n$-hexane and dichloromethane xerogels. This is indicative of a more compact assembly in this polar solvent. Due to the lack of a chromophore in $\mathbf{1}$, it was not possible to use circular dichroism to prove the helicoidal nature of the fibrillar network, but images suggest that, at least in dichloromethane, the one dimensional self-assembly is directed helicoidally by the inherently asymmetric steroid molecule.

To gain a better insight into the packing of the material, X-ray powder diffractograms (XRPD) of xerogels from dichloromethane (DCM) and $n$-hexane were performed (Figure 5). The X-ray pattern of the xerogel of $\mathbf{1}$ from $n$-hexane showed an intense scattering peak at $d=35.0 \AA$ with a small shoulder at $27.6 \AA$ and two smaller and broader peaks at $d=6.0$ and $5.0 \AA$ (Figure 5a). The larger $d$ value can be associated to the repeating distance of the aggregates. In the case of the xerogel from DCM this peak appears at $d=29.4 \AA$, and the generally less sharp peaks indicate a less ordered self-assembly (Figure 5b). As shown in Figure 6, the scattering peak at $d=35 \AA$ of the $n$-hexane gel perfectly correlates with the distance of two molecules of $\mathbf{1}$ arranged head-to-head with

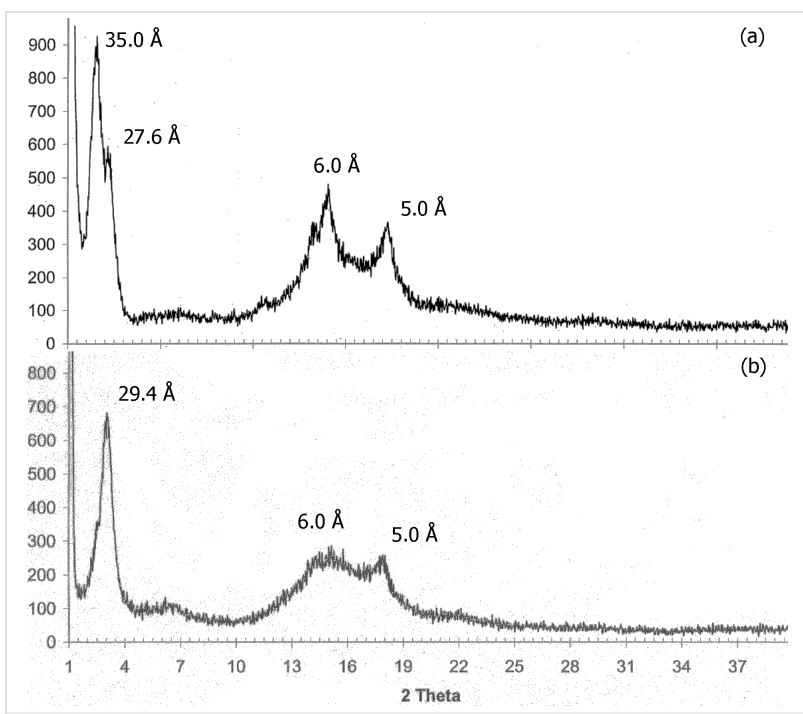

Figure 5: Powder X-ray diffraction pattern of the xerogels of $\mathbf{1}$ from a) $n$-hexane and b) dichloromethane.
A

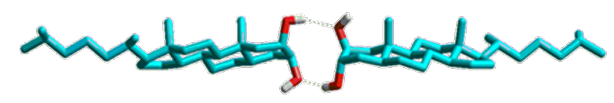

$36.6 \AA$
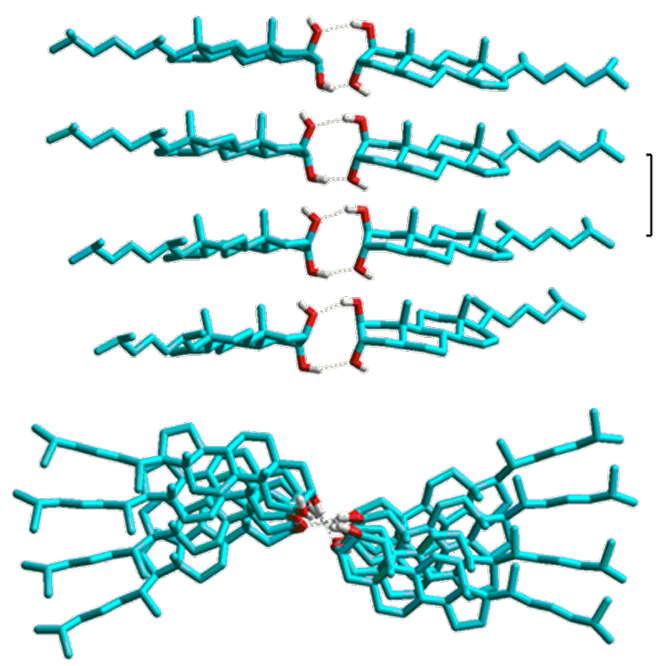

B
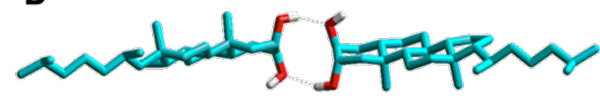

hydrogen bond $2.3 \AA$

$6.3 \AA$
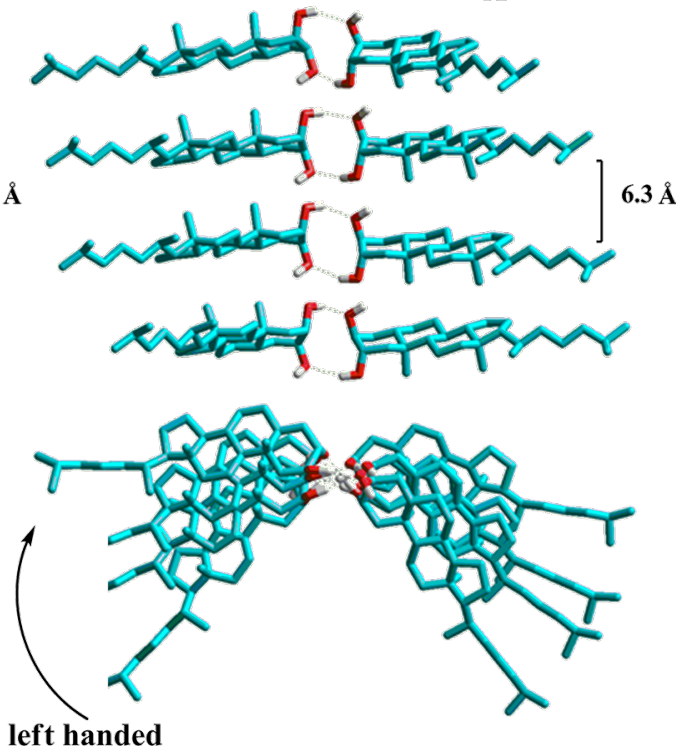

Figure 6: Self-assembly models proposed for LMOG 1, only the left handed helix is shown, head to head hydrogen bonds are shown in dotted lines, hydrogen atoms have been removed for clarity. System A: hydrogen bonds at $\mathrm{C} 2-\mathrm{OH} / \mathrm{C2}^{\prime}-\mathrm{OH}$ and $\mathrm{C} 3-\mathrm{OH} / \mathrm{C} 3^{\prime}-\mathrm{OH}$; system B: $\mathrm{C} 2-\mathrm{OH} / \mathrm{C}^{\prime}-\mathrm{OH}$ and $\mathrm{C} 2{ }^{\prime}-\mathrm{OH} / \mathrm{C} 3-\mathrm{OH}$. 
completely elongated side chains. Taking this into account the specific molecular packing of gels of $\mathbf{1}$ may be understood from its molecular structure itself. Steroid $\mathbf{1}$ is a typical amphiphilic compound in which the dihydroxy moiety defines a hydrophilic head while the rest of the skeleton (tail) is hydrophobic. The amphiphilic property causes molecules to aggregate into structures that avoid the unfavorable head-to-tail contact, and so, head-to-head and tail-to-tail contacts are directing the selfassembly. In more polar solvents such as DCM, the side chain of the steroid is not fully elongated to avoid the interaction of DCM molecules with the polar head. This is evidenced by the shift of $d$ from $35.0 \AA$ ( $n$-hexane) to $29.4 \AA$ (DCM). On the other hand, the spatial orientation of the hydroxy groups is critical since only steroid $\mathbf{1}$, with both hydroxy groups at axial positions, has the ability of a long range self-assembly to promote gelation. To get an insight of the necessary molecular spatial requirements for the 2,3-dihydroxy moiety to reach an optimal hydrogen bonding, we studied the possible mode of selfassembly of the four isomers by a molecular modeling simulation in vacuum (semiempirical AM1).

A conformational study of a single molecule of LMOG 1 (semiempirical, AM1) showed a distance of $18.0 \AA$ for the molecule with the elongated side chain (Figure 6) suggesting a repetitive unit involving a head-to-head self-assembly between two molecules, as explained above. Next, we minimized different modes of head-to-head interactions and found two possible arrangements with minimal energies. In the first one, both molecules interact with the $\alpha$ faces of the steroids pointing to the same side (Figure 6, system A). In the second case the $\alpha$ faces are oriented to opposite sides (Figure 6, system B). Both dimers were similar in energy with stabilizations of 9.7 and $10.0 \mathrm{kcal} / \mathrm{mol}$ compared to the isolated molecules. This stabilization energy arises from the two intermolecular hydrogen bonds between the hydroxy groups with a $\mathrm{H}-\mathrm{OH}$ distance of $2.4 \AA$. We then analyzed the one-dimensional arrangements of the dimers described above by an $\alpha$ - and $\beta$-helix self-assembly. We placed the dimers in a way so that the central hydrophilic zones can interact with each other with a distance of $2.7 \AA$ between the hydroxy groups (to allow hydrogen bond interactions) and a rotation angle around the hydrogen bond axis of \pm 18 degrees (corresponding to leftand right-handed helices, respectively). After minimization, the left handed assemblies were slightly more stable than the right handed, but with no significant differences $(0.2-0.3 \mathrm{kcal} / \mathrm{mol})$. For this reason we will only discuss the energy of the lefthanded systems. The stabilization energy for each dimer-dimer interface for the $\beta$-octamer $\mathbf{A}$ was $8.1 \mathrm{kcal} / \mathrm{mol}$ and for the $\beta$-octamer $\mathbf{B} 8.3 \mathrm{kcal} / \mathrm{mol}$. This stabilization between the dimers arises from hydrogen bonding between the dimers with $\mathrm{H}-\mathrm{OH}$ distances of about $2.8 \AA$, and van der Waals interactions between the hydrocarbonated skeletons of the steroid.
A structural analysis of the steroids 2, 3 and 4, with hydroxy groups at equatorial positions, showed that angles and distances between the hydroxy groups are inadequate to give the intermolecular hydrogen bonding necessary for the one-dimensional self-assembly. These results led to the conclusion that for LMOG 1, the arrangements proposed are valid modes of selfassembly in agreement with the experimental results. Both, leftand right-handed self-assembled systems, are stable at the conditions of the calculations and this stabilization arises from the multiple hydrogen bonds that are only allowed for the transdiaxial dihydroxy system present in organogelator 1. As mentioned before, the existence of two clearly differentiated gelation zones in the HSP plot is indicative for a difference in the molecular packing of the gel fibers in non-polar and nonprotic polar solvents. In the last case the packing is tight enough to prevent the polar solvent molecules to interact with the polar head of the steroid breaking the hydrogen bonds that lead to the SAFIN.

\section{Templated preparation of silica nanoparticles}

Even though LMOG 1 does not gelate tetraethoxysilane (TEOS), in-situ sol-gel polymerization experiments were performed with dioxane and dichloromethane gels containing $16.6 \%$ of TEOS with benzylamine as a catalyst. The morphology of the nanostructured silica obtained was analyzed by SEM microscopy (Figure 7). A first polymerization attempt using $3 \mu \mathrm{L}$ of catalyst and $15 \mu \mathrm{L}$ of water showed a mixture of amorphous silica with nanotubes in a $3 / 2$ ratio reflecting a partial template polymerization process (Figure $7 \mathrm{a}$ ). Since high reaction rates usually disfavor the template process for the sol-gel polymerization of TEOS, we decided to slow down the reaction rate by lowering the water concentration and the catalyst load. In the first case (Figure 7b) a highly amorphous material was observed, while lowering the benzylamine load to a third rendered only nanotubes of silica with external diameters between 50 and $175 \mathrm{~nm}$ and lengths of several micrometers (Figure 7c,d). Lowering the catalyst load down to a tenth rendered a higher amount of amorphous material. To assess the role of the fibrillar network in the templating process we repeated the experiment under the same conditions, in which nanotubes were obtained, but in absence of gelator. In this case only amorphous silica was observed by SEM microscopy of the product (see Supporting Information File 1), which proves the directing role of the fibers during the polymerization of TEOS. Next we performed the polymerization of TEOS in dichloromethane gels to confirm the helicoidally nature of the fibers by templation. The SEM images of the product obtained showed spherical nanoparticles of irregular size (see Supporting Information File 1). In this solvent the templated sol-gel polymerization failed, but the formation of spherical particles indicates some control effect on the growth of the silica nucleus by the 


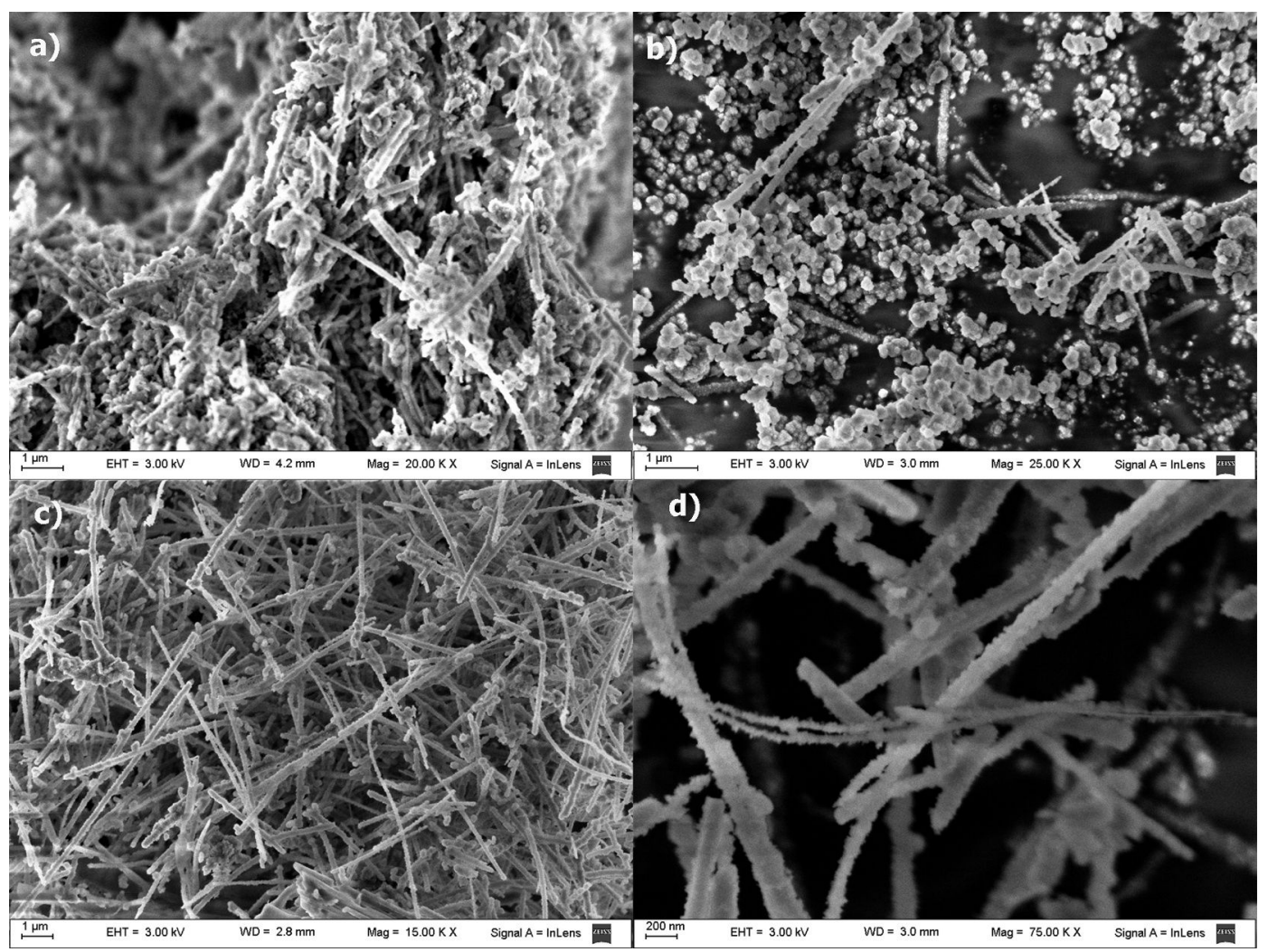

Figure 7: SEM images of nanostructured silica obtained from gels of LMOG 1 under the following conditions: $0.5 \mathrm{~mL}$ of dioxane, $0.1 \mathrm{~mL}$ TEOS, $35 \mathrm{mg}$ of 1 , and (a) $3 \mu \mathrm{L}$ of benzylamine and $15 \mu \mathrm{L}$ of water, (b) $3 \mu \mathrm{L}$ of benzylamine and $3 \mu \mathrm{L}$ of water (c,d) $1 \mu \mathrm{L}$ of benzylamine and $15 \mu \mathrm{L}$ of water.

fibrillar network of the gel. Tubular nanostructured materials, such as these presented here, may offer alternatives over spherical nanoparticles for some biomedical and biotechnological applications $[30,31]$.

\section{Conclusion}

In summary, we have studied the organogelating behavior of the four stereoisomers of the structurally simple 2,3-dihydroxycholestane. The theoretical and experimental results on the four stereoisomers indicate that the trans-diaxial orientation of the hydroxy groups on this supramolecular synthon is essential for the gelating property. Only the isomer with the trans-diaxial dihydroxy group had the ability to gelate a wide variety of organic solvents and to be a superorganogelator for hydrocarbons with a minimal concentration for gelation of $0.04 \mathrm{wt} \%$. From the analysis of the solvent parameters we conclude that the Hansen solubility parameter (HSP) approach is more suitable to study the solvent-gelation relationship than the solvatochromic Kamlet-Taft scale. The HSP analysis showed two gelating zones indicative of different packing in polar and nonpolar solvents. In contrast to the results obtained by Boutellier et al., it was not possible to define a gelation sphere in either zone, but a qualitative analysis showed that predictions are still possible for this complex system. The FTIR, XRPD, and semiempirical molecular modeling studies allowed us to propose a packing mode in which the amphiphilic steroid self-assembles in a head-to-head mode through two hydrogen bonds between the dihydroxylic system. The resulting dimers can then form 1D aggregates, in which multiple hydrogen bonding plays an important role together with van der Waals interaction stabilization. In case of polar solvents, capable of hydrogen bond formation, the packing of LMOG 1 is tighter in order to prevent the solvent molecules to interact with the polar head of the steroids, which would break the self-assembled fibrillar network. Finally, the dioxane gel was successfully used as template to grow silica nanotubes through sol-gel polymerization of TEOS under basic catalysis. We conclude that the success of the template on dioxane strongly depends on the catalyst load. Usually the structural motif or element enabling the more efficient transcription for template synthesis of inorganic oxides involves a covalently attached positive charge. For neutral organogelator 1, electrostatic or hydrogen-bond interactions between the intermediate anionic silicate species and the fibrillar network may be proposed as the only driving force directing the template. Such 
nanostructured materials may have biomedical and biotechnological applications that offer alternatives over spherical nanoparticles. Among the solvents gelled by steroid 1, styrene and methyl acrylate offer great potential in the preparation of mesoporous polymers. We are currently exploring these materials and their potential applications.

\section{Experimental Materials}

Cholesterol (94\%) was purchased from Sigma-Aldrich. $n$-Hexane, ethyl acetate, dichloromethane and THF were fractionally distilled, and the remaining solvents were used as supplied by the manufactures.

\section{Methods}

Synthesis: $2 \beta, 3 \alpha$-dihydroxycholestane (1), $2 \alpha, 3 \beta$-dihydroxy-

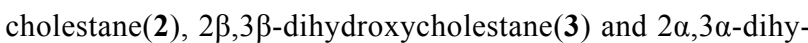
droxycholestane (4) were prepared from cholesterol following the procedures described in the literature [27]. The identities of compounds 1, 3 and $\mathbf{4}$ were confirmed by comparing the NMR spectra with those found in the literature. ${ }^{13} \mathrm{C}$ NMR spectra of compound 2 did not match the literature data $[27,28]$ and was completely characterized to confirm its identity concluding that the ${ }^{13} \mathrm{C}$ NMR data reported in literature was mistaken (see Supporting Information File 1).

Gelation Tests: The test were carried out in a similar manner as described in [25]. The gelation ability was investigated by a typical test tube experiment. A mixture of a defined amount of gelator and a volume of the solvent $(10 \% \mathrm{wt} / \mathrm{v})$ in a closed flask was heated and shaken until the solid was dissolved and then slowly cooled to room temperature. If a stable gel was observed after inversion of the flask, it was considered a gel $(\mathrm{G})$. When gelation was not observed at room temperature, the sample was cooled at $5{ }^{\circ} \mathrm{C}$. The critical concentration for gelation (CCG) was determined by subsequent dilution of the original organogel followed by a heating-cooling process until gel formation was not observed at room temperature $\left(20^{\circ} \mathrm{C}\right)$

The reversible gel-sol transition temperatures $\left(T_{\mathrm{g}}\right)$ were measured using the classical inverted tube method [32].

Phase-selective gelation experiment: Compound 1 ( $8 \mathrm{mg})$ was added to a flask with a mixture of $1 \mathrm{~mL}$ of dichloromethane and $1 \mathrm{~mL}$ of water, the flask was closed, shaken and heated until the solid was dissolved. Then, the solution was cooled and left at room temperature. After ca.15 min the dichloromethane phase became a gel and the water layer was still fluid.

Xerogel preparation: The preparation was carried out in a similar manner as described in [25]. The xerogels were prepared by cooling the gels in a bath at $-90{ }^{\circ} \mathrm{C}$, evaporating the solvent under high vacuum over $6 \mathrm{~h}$ and then slowly letting the gels get to room temperature under vacuum.

FTIR measurements: The measurements were carried in a similar manner as described in [25]. Fourier transform infrared (FTIR) measurements of the solution and the gels of 1 were performed on a Nicolet Magna IR 550 FTIR spectrometer in a demountable liquid cell with two $\mathrm{NaBr}$ disks, $32 \mathrm{~mm}$ in diameter and a $0.5 \mathrm{~mm}$ thick Teflon spacer. For the dichloromethane gel, a warm solution of $1(0.25 \mathrm{wt} \%)$ was injected into the cell and allowed to cool down for $10 \mathrm{~min}$ at room temperature before measuring the spectra.

X-ray powder diffraction measurements: Diffraction patterns were obtained by using a Siemens D5000 diffractometer with $\mathrm{Cu} \mathrm{K} \alpha$ radiation $(\lambda=1.54056 \AA)$, the stepsize was $0.025^{\circ}$ with a measurement time of $6 \mathrm{~s}$ per step.

Scanning electron microscopy: SEM measurements were performed in a similar manner as described in [25]. SEM pictures of the xerogel and silica nanoparticles were taken on a Carl Zeiss NTS SUPRA 40FEG scanning electron microscope. A small portion of the solid sample (xerogel or silica) was attached to the holder by using a conductive adhesive carbon tape. Prior to examination the xerogels were coated with a thin layer of gold.

Sol-Gel polymerization of TEOS in dioxane: Compound 1 (35 mg) was dissolved by heating and shaking in dioxane $(0.5 \mathrm{~mL})$ and TEOS $(0.1 \mathrm{~mL})$ with an addition of benzylamine $(0.1-3.0 \mu \mathrm{L})$ and water $(3-15 \mu \mathrm{L})$. The solution was cooled down to room temperature until gelation was observed and then left at room temperature for 6 days. Subsequently, the sample was diluted in dichloromethane, the solid was centrifuged, and washed once with dichloromethane. The silica was heated at $200{ }^{\circ} \mathrm{C}$ for $2 \mathrm{~h}$ and $600{ }^{\circ} \mathrm{C}$ for $4 \mathrm{~h}$ in air.

Molecular modeling experiment: These experiments were conducted in a similar manner as described in [25]. The computational experiments were performed with HyperChem 8.0.4, semiempirical optimization, AM1 method in vacuum. Algorithm: Fletcher-Reeves. Termination condition, RMS gradient: $0.05 \mathrm{kcal} /(\AA \cdot \mathrm{mol})$. No bond or distance restrictions were imposed. The interaction energies were estimated from the difference between the heats of formation of the different arrangements divided by the number of molecule-molecule interfaces. To have an insight in the stabilization energy of the 1D self-assembled models proposed we carried out the following experiments: Experiment I: an isolated molecule of LMOG 1 was minimized using the above conditions; 
$\Delta H_{\mathrm{f}}=-201.46 \mathrm{kcal} / \mathrm{mol}$. Experiment II: head-to-head dimers of LMOG 1 were minimized, the molecules were placed facing the hydroxy groups at a distance typical for hydrogen bonds: Dimeric system A: $\mathrm{C} 2-\mathrm{OH} / \mathrm{C}^{\prime}{ }^{\prime}-\mathrm{OH}$ and $\mathrm{C} 3-\mathrm{OH} / \mathrm{C}^{\prime}{ }^{\prime}-\mathrm{OH}$; $\Delta \mathrm{H}_{\mathrm{f}}=-412.60 \mathrm{kcal} / \mathrm{mol}$. Dimeric system B: C2-OH/C3'-OH and $\mathrm{C}^{\prime}{ }^{\prime}-\mathrm{OH} / \mathrm{C} 3-\mathrm{OH} ; \Delta H_{\mathrm{f}}=-412.91 \mathrm{kcal} / \mathrm{mol}$. Experiment III: four dimers from experiments II were placed in a 1D arrangement facing the $\alpha$ - and $\beta$-faces of the steroids with a separation of about $6 \AA$ between the steroid skeleton, and a rotation angle around the hydrogen bond axis of $+18^{\circ}$ and $-18^{\circ}(\alpha-$ and $\beta$-helix). No bond or distance restrictions were imposed. The right and left handed helix of systems $\mathbf{A}$ and $\mathbf{B}$ gave similar heats of formation with no significant differences. Octameric system A (left handed helix): $\Delta H_{\mathrm{f}}=-1674.52 \mathrm{kcal} / \mathrm{mol}$; octameric system B (left handed helix): $\Delta H_{\mathrm{f}}=-1676.62 \mathrm{kcal} / \mathrm{mol}$. The stabilization energy for the head-to-head interaction in each molecule-molecule interface was estimated from the difference between the heats of formation of the dimers and the isolated molecules. The stabilization energy for the dimer-dimer interaction in the one dimensional arrangement interface was estimated from the difference between the heats of formation of the octamers and the dimers.

\section{Supporting Information}

Supporting Information features additional experimental data, i.e. characterization data of steroid 2, SEM images, $T_{\mathrm{g}}$-vs-concentration plots, FTIR spectra, and HSP plots.

\section{Supporting Information File 1}

Additional experimental data.

[http://www.beilstein-journals.org/bjoc/content/

supplementary/1860-5397-9-213-S1.pdf]

\section{Acknowledgements}

The authors are grateful to ANPCyT (Argentina), CONICET (Argentina) and the University of Buenos Aires for financial support.

\section{References}

1. Shahmburo, A.; Biewer, M. C. Chem. Mater. 2002, 14, 3745-3750. doi:10.1021/cm020421a

2. Llusar, M.; Sanchez, C. Chem. Mater. 2008, 20, 782-820. doi:10.1021/cm702141e

3. Wang, X.; Zhou, L.; Wang, H.; Luo, Q.; Xu, J.; Liu, J. J. Colloid Interface Sci. 2011, 353, 412-419. doi:10.1016/j.jcis.2010.09.089

4. Prathap, A.; Sureshman, K. M. Chem. Commun. 2012, 48, 5250-5252. doi:10.1039/c2cc31631e
5. Shibata, Y.; Kato, T.; Kado, T.; Shiratuchi, R.; Takashima, R.; Kaneto, W.; Hayase, S. Chem. Commun. 2003, 2730-2731. doi:10.1039/b305368g

6. Ajayaghosh, A.; Praveen, V. K.; Vijayakumar, C. Chem. Soc. Rev. 2008, 37, 109-122. doi:10.1039/b704456a

7. Li, Y.; Liu, J.; Du, G.; Yan, H.; Wang, H.; Zhang, H.; An, W.; Zhao, W.; Sun, T.; Xin, F.; Kong, L.; Li, Y.; Hao, A.; Hao, J. J. Phys. Chem. B 2010, 114, 10321-10326. doi:10.1021/jp1017373

8. Murata, K.; Aoki, M.; Suzuki, T.; Harada, T.; Kawabata, H.; Komori, T.; Ohseto, F.; Ueda, K.; Shinkai, S. J. Am. Chem. Soc. 1994, 116, 6664-6676. doi:10.1021/ja00094a023

9. Yu, X.; Liu, Q.; Wu, J.; Zhang, M.; Cao, X.; Zhang, S.; Wang, Q.; Chen, L.; Yi, T. Chem.-Eur. J. 2010, 16, 9099-9106. doi:10.1002/chem.201000187

10. Maitra, U.; Chakrabarty, A. Beilstein J. Org. Chem. 2011, 7, 304-309. doi:10.3762/bjoc. 7.40

11. Chen, J.; Wu, W.; Mc Neil, A. J. Chem. Commun. 2012, 48 , 7310-7312. doi:10.1039/c2cc33486k

12. Díaz Díaz, D.; Kühbeck, D.; Koopmans, R. J. Chem. Soc. Rev. 2011, 40, 427-448. doi:10.1039/c005401c

13. Weiss, R. G.; Terech, P. Molecular Gels: Materials with Self-Assembled Fibrillar Networks; Springer: The Netherlands, 2006.

14. Desiraju, G. R. Angew. Chem., Int. Ed. Engl. 1995, 34, 2311-2327. doi:10.1002/anie.199523111

15. Sahoo, P.; Chakraborty, I.; Dastidar, P. Soft Matter 2012, 8, 2595-2598. doi:10.1039/c2sm06957a

16. Adalder, T. K.; Adarsh, N. N.; Sankolli, R.; Dastidar, P. Beilstein J. Org. Chem. 2010, 6, 848-858. doi:10.3762/bjoc.6.100

17. Hirst, A. R.; Coates, I. A.; Boucheteau, T. R.; Miravet, J. F.; Escuder, B.; Castelletto, V.; Hamley, I. W.; Smith, D. K. J. Am. Chem. Soc. 2008, 130, 9113-9121. doi:10.1021/ja801804c

18. Raynal, M.; Bouteiller, L. Chem. Commun. 2011, 47, 8271-8273. doi:10.1039/c1cc13244j

19. Gao, J.; Wu, S.; Rogers, M. A. J. Mater. Chem. 2012, 22, 12651-12658. doi:10.1039/c2jm32056h

20. Edwards, W.; Lagadec, C. A.; Smith, D. K. Soft Matter 2011, 7, 110-117. doi:10.1039/c0sm00843e

21. Löfman, M.; Koivukorpi, J.; Noponen, V.; Salo, H.; Sievänen, E. J. Colloid Interface Sci. 2011, 360, 633-644. doi:10.1016/j.jcis.2011.04.112

22. Zinic, M.; Vögtle, F.; Fages, F. In Gelation with Small Molecules; Fages, F., Ed.; Springer Verlag: Berlin, Germany, 2005; pp 39-63.

23. Lin, Y. C.; Kachar, B.; Weiss, R. G. J. Am. Chem. Soc. 1989, 111, 5542-5551. doi:10.1021/ja00197a005

24. Svobodová, H.; Noponen, V.; Kolehmainen, E.; Sievänen, E. RSC Adv. 2012, 2, 4985-5007. doi:10.1039/c2ra01343f

25. Edelsztein, V. C.; Burton, G.; Di Chenna, P. H. Tetrahedron 2010, 66, 2162-2167. doi:10.1016/j.tet.2010.01.065

26. Edelsztein, V. C.; Jares-Erijman, E. A.; Müllen, K.; Di Chenna, P. H.; Spagnuolo, C. C. J. Mater. Chem. 2012, 22, 21857-21861. doi:10.1039/c2jm34891h

27. Cruz Silva, M. M.; Riva, S.; Sá e Melo, M. L. Tetrahedron 2005, 61, 3065-3073. doi:10.1016/j.tet.2005.01.104

28. Jursic, B. S.; Upadhyay, S. K.; Creech, C. C.; Neumann, D. M. Bioorg. Med. Chem. Lett. 2010, 24, 7372-7375. doi:10.1016/j.bmcl.2010.10.044

29. Kamlet, M. J.; Abboud, J. L. M.; Abraham, M. H.; Taft, R. W. J. Org. Chem. 1983, 48, 2877-2887. doi:10.1021/jo00165a018

30. Martin, C. R.; Kohli, P. Nat. Rev. Drug Discovery 2003, 2, 29-37. doi:10.1038/nrd988 
31. Bae, S. W.; Tan, W.; Hong, J.-I. Chem. Commun. 2012, 48 , 2270-2282. doi:10.1039/c2cc16306c

32. Raghavan, S. R.; Cipriano, B. H. Gel formation: Phase Diagrams using table top Rheology and Calorimetry. In Molecular Gels: Materials with Self-Assembled Fibrillar Networks; Weiss, R. G.; Terech, P., Eds.; Springer Verlag: The Netherlands, 2006.

\section{License and Terms}

This is an Open Access article under the terms of the Creative Commons Attribution License

(http://creativecommons.org/licenses/by/2.0), which permits unrestricted use, distribution, and reproduction in any medium, provided the original work is properly cited.

The license is subject to the Beilstein Journal of Organic Chemistry terms and conditions:

(http://www.beilstein-journals.org/bjoc)

The definitive version of this article is the electronic one which can be found at: doi:10.3762/bjoc. 9.213 